\title{
The Feasibility Study on Biodrying for Solid Refuse Fuel (SRF) of Municipal Sewage Sludge
}

\author{
Minah Oh ${ }^{1}$, Jeong Won An ${ }^{1}$, Seungjin Oh${ }^{1}$, Hwa-Soo Oh$^{2}$, Jai-Young Lee ${ }^{1}$ \\ ${ }^{1}$ Dept. of environmental engineering, The University of Seoul \\ 163 Seoulsiripdae-rom Dongdaemun-gu, Seoul, South Korea \\ minawizz@uos.ac.kr; jw3654@gmail.com; ohpromote@gmail.com; leejy@uos.ac.kr \\ ${ }^{2}$ Tobang E\&E \\ A-306, 410 Jeongseojin-ro, Seo-gu, Inchoen, South Korea \\ ohhsoo@hanmail.net
}

\section{Extended Abstract}

In Korea, the landfilling of sewage sludge directly was prohibited and London agreement which forbid ocean dumping was conducted at 2012. Thus, adequate treatment method about sewage sludge is required [1]. Meanwhile, waste treatment policy paradigm is changing quickly organic waste to recycling and fueling because of Activation of Clean Development Mechanism (CDM) Project under International Climate Change Convention. However, it spends higher operating costs and longer time to drying for recycling and fueling that organic waste which contains high moisture as sewage sludge [2]. In this study, aerobic microorganisms were applied as a pre-treatment to produce sewage sludge into a solid fuel, and the possibility of moisture contents reduction by exothermic reaction was evaluated [3]. 35L cylindrical acrylic reactor (Figure 1) was applied for aerobic pre-treatment test. Digested sewage sludge was collected at the Waste Water Treatment Plant (WWTP) located in Gangnam-gu, Seoul. For the aerobic reaction, $100 \mathrm{ml} / \mathrm{min} \cdot \mathrm{kg}$ air was injected by blower, at lower and middle part of the reactor and stirred by motor at the top of the reactor. The aerobic pre-treatment test were conducted for 14days in total, and the moisture contents were gradually decreased which was $79 \%$ at the initial operation. At the end of the operation, moisture contents were investigated $63 \%$ that was decreased amount of $16 \%$ than primary value. Especially, temperature of reactor was much higher at 6 9 days than other days, as a result moisture contents were decreased significantly. So that maintaining high temperature is a major objective in this research. Especially, the decrease of moisture content was observed to be significant over $6 \sim 8$ days when the temperature in the reactor was relatively high, and it was confirmed that maintenance of the high temperature section was the most important purpose of the study. The initial lower heat values (LHV) of sewage sludge is $3,210 \mathrm{kcal} / \mathrm{kg}$, at the end of the process, LHV was decreased to 2,833 $\mathrm{kcal} / \mathrm{kg}$. It because aerobic microorganisms degrade organic matter in sewage sludge (Figure 2). In this study, additional test were conducted by varying the amount of air injection which flow rate was $200 \mathrm{ml} / \mathrm{min} \cdot \mathrm{kg}$, progressed at $400 \mathrm{ml} /$ min $\cdot \mathrm{kg}$ with 2 times and 4 times the initial test, as a result the moisture content which reactor with air injected $200 \mathrm{ml} /$ min $\cdot \mathrm{kg}$ was most dramatically reduced (Figure 3). Through this study, we confirmed effect of moisture content reduction in sewage sludge using aerobic pre-treatment. 


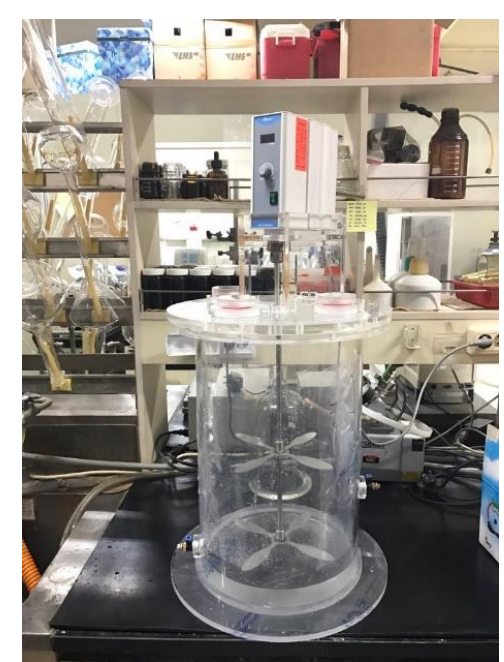

Fig. 1: 35L cylindrical acrylic reactor.
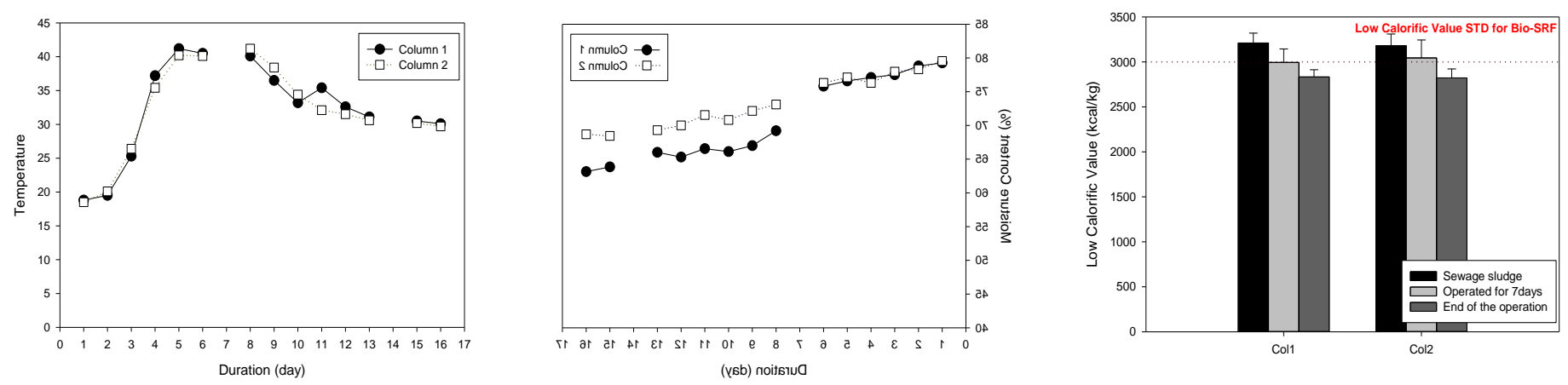

Fig. 2: Changes of temperature and moisture content during Bio-drying test.
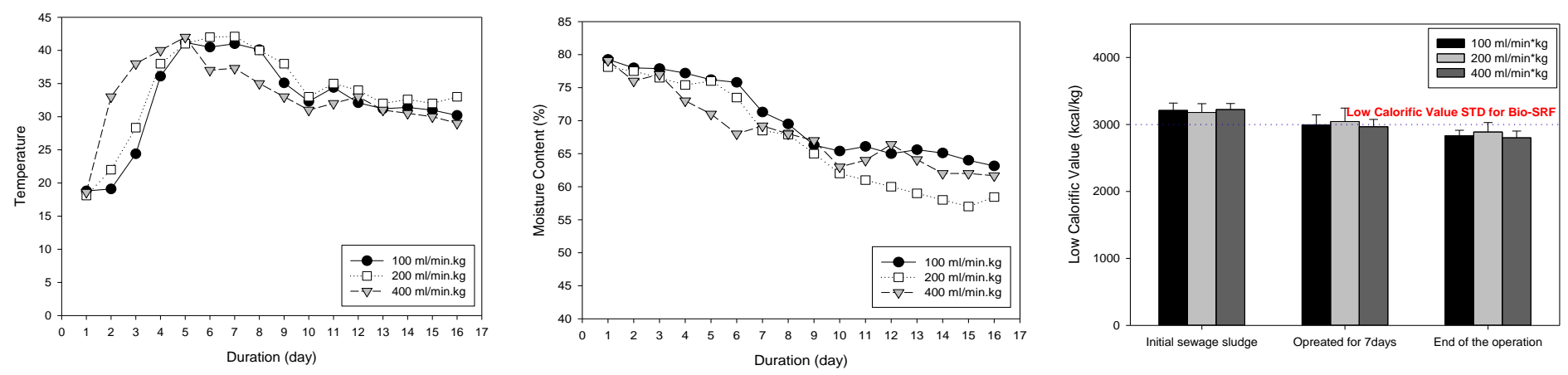

Fig. 3: Changes of temperature and moisture content related to aeration rate on Bio-drying test.

\section{Acknowledgements}

This work was funded by the Small and Medium Business Administration in Korea (Project\# S2405744).

\section{References}

[1] J. U. Jung, "Study of bio-drying process for the production of mixed bio-SRF of sewage sludge with coffee ground," M.S. Thesis, Andong National University, 2014.

[2] H. H. Lee, "Effect of air-flow rate and directions on water removal of aerated static pile bio-drying process," M.S. Thesis, The University of Seoul, 2017.

[3] L. Cai, D. Gao, T. B. Chen, H. T. Liu, G. D. Zheng and Q. W. Yang, "Moisture variation associated with water input and evaporation during sewage sludge bio-drying," Bioresource Technology, vol. 117, pp. 13-19, 2012. 